\title{
A double-blinded randomised dietary supplement crossover trial design to investigate the short-term influence of medium chain fatty acid (MCT) supplement on canine idiopathic epilepsy: study protocol
}

Benjamin Andreas Berk ${ }^{1,2^{*}}$ (D), Rowena Mary Anne Packer ${ }^{1}$, Tsz Hong Law $^{1}$, Annette Wessmann ${ }^{3}$, Andrea Bathen-Nöthen ${ }^{4}$, Tarja Susanna Jokinen ${ }^{5}$, Anna Knebel ${ }^{6}$, Andrea Tipold ${ }^{6}$, Ludovic Pelligand ${ }^{7}$ and Holger Andreas Volk ${ }^{1,6}$

\begin{abstract}
Background: Epilepsy is the most common brain disease in dogs. Recently, diets have been reported to have a positive impact on seizure activity and behaviour in various species including dogs with idiopathic epilepsy (IE). Historically, classic high fat ketogenic diets (KD) and medium chain triglycerides (MCT) KD have been successfully used to manage drug-resistant epilepsy. Similarly, an MCT enriched diet has been shown to improve seizure control and behavioural comorbidities in some dogs with IE. However, it is unknown whether an MCT dietary supplement (DS) may provide similar positive effects.
\end{abstract}

Methods: A 6-month prospective, randomised, double-blinded, placebo-controlled, crossover, multicentre dietary trial is designed comparing a $9 \%$ metabolic energy based calculated medium-chain triglyceride (MCT) oil supplement to a conventional 'control' DS. Only dogs which will have an International Veterinary Epilepsy Task Force Tier II level like diagnosis of IE which satisfied the following inclusion criteria are included: age between 6 months and $\leq 12$ years; weighing between 4 and $\leq 65 \mathrm{~kg}$; unremarkable interictal neurological examinations; no clinically significant findings on routine laboratory diagnostics; unremarkable brain MRI scan; have had at least 3 seizures in the previous 3 months prior to enrolment; treated with at least one ASD and being classified as resistant. All dogs are fed initially for $90 \pm 2$ days with either the control oil or the MCT oil alongside their normal diet, followed by $97 \pm 2$ days with the other supplement including a 7-day washout period. Overall, the aim is to recruit thirty-six patients at five different centres and to investigate the effect of MCTs as DS on seizure activity, tolerability, behavioural comorbidities and quality of life (QoL).

(Continued on next page)

\footnotetext{
* Correspondence: bberk@rvc.ac.uk

${ }^{1}$ Department of Clinical Science and Services (CSS), Royal Veterinary College, Hatfield, UK

${ }^{2}$ Tierarztpraxis Strassenheim: BrainCheck.Pet, Ortsstraße, Mannheim, Germany

Full list of author information is available at the end of the article
}

(c) The Author(s). 2019 Open Access This article is distributed under the terms of the Creative Commons Attribution 4.0 International License (http://creativecommons.org/licenses/by/4.0/), which permits unrestricted use, distribution, and reproduction in any medium, provided you give appropriate credit to the original author(s) and the source, provide a link to the Creative Commons license, and indicate if changes were made. The Creative Commons Public Domain Dedication waiver (http://creativecommons.org/publicdomain/zero/1.0/) applies to the data made available in this article, unless otherwise stated. 
(Continued from previous page)

Discussion: Dietary interventions are rarely studied in a standardised form in veterinary medicine. The background diet, the cohort of animals and ASD received is standardised in this prospective diet trial to ensure representative data about the potential effect of MCT DS. If the study data confirms former findings, this would provide further evidence for the efficacy of MCTs as a management option for canine epilepsy. This publication should offer a repository of trial conditions and variable description with forecasted statistical analysis.

Keywords: Veterinary neurology, Epilepsy, Diet, Medium-chain triglyceride, Medium-chain fatty acids, Seizure

\section{Background}

Epilepsy, defined by the disposition to having seizures, is the most common chronic neurological disease in humans and dogs $[1,2]$. Within the UK alone, epidemiological studies have revealed that an estimated $0.6 \%$ of dogs are affected by idiopathic epilepsy (IE) [3]. Epilepsy is a major risk to health and welfare in dogs. This neurological disorder has been associated with physical, cognitive and neurobehavioural comorbidities and increased risk of premature death [4].

Currently, pharmacotherapy with seizure suppressing drugs ("anti-epileptic drugs "or "anti-seizure drugs" (ASD)) represents the most important form of treatment in veterinary medicine. Epileptic patients are usually treated on a chronic, often life-long basis [4]. However, permanent ASD administration is characterised by determining the fine balance between benefits and disadvantages of ASDs. The seizure-suppressing effects of ASDs might be outweighed by unfavourable side effects including polyphagia, polydipsia, polyuria, restlessness, lethargy or ataxia [5]. Owners of dogs with IE are often worried about the level of sedation and ataxia induced by ASDs, their dog's seizure frequency, and polypharmacy affecting their own and their pets' quality of life (QoL) [6].

Furthermore, despite well-established ASD therapies, over two thirds of dogs with epilepsy continue to experience seizures in the long-term and fail to achieve total epilepsy control [7-9]. Up to 30\% of these dogs treated with two or more ASDs continue to experience seizures with a less than $50 \%$ reduction in seizure frequency $[8,10-12]$. This emphasises the need for new therapeutic interventions to improve the QoL of dogs with IE [7, 13-15].

Various diets, especially ketogenic diets (KD), have been shown to reduce frequency and severity of seizures in humans with epilepsy and in rodent models of epilepsy [14, 16-21]. Lifestyle variables, such as the diet of dogs with IE, have also been recognised both anecdotally and in published literature as having an impact upon seizure activity and behaviour [22-25]. For example, a trial of eight dogs with epilepsy treated with an exclusion diet was reported in 2004 with seven dogs achieving a reduction in seizure frequency [26]. Unfortunately, the exact mechanism leading to the anti-epileptic and/or anti-seizure properties of KDs has not yet been fully elucidated, highlighting the need for more research, especially in veterinary medicine, to confirm the effects of diet in dogs with IE.

The ketogenic diet was first used in human epilepsy to mimic the metabolic state of fasting, as fasting was shown to have anti-seizure effects [27]. Under conditions of low carbohydrate intake, such as fasting, the liver metabolises triglycerides and releases fatty acids and subsequent ketone bodies into blood circulation, a process known as ketosis [28]. The ketone bodies replace glucose as the main source of energy in the brain [29]. A rise in brain ketones has been suggested to evoke anti-epileptic properties [30]. The ketone body Beta-hydroxybutric acid (BHB) has been shown to reduce seizure-like neuronal activity and is thought to be mediated via $K_{\text {ATP }}$ channel activity and inhibiting $\mathrm{GABA}_{\mathrm{B}}$ signalling in a Drosophila in-vitro model [31]. Similarly, intraperitoneal injection of BHB led to increased BHB serum concentrations and prolonged onset of seizures in a rat seizure model accompanied with neuroprotective effects observed in histopathology [32]. Other, newer in-vitro studies assumed BHB as having neuroprotective function by direct gene regulation and promotion of brain-derived neurotrophic factor (BDNF) expression [33]. Apart from BHB, other in-vitro and in-vivo studies in rodent epilepsy models on other ketone bodies, such as acetone or acetoacetate, also indicated anticonvulsant action, ion channel interaction and potential synergistic role in ketogenic diets [34-38]. Many studies have reported the anti-seizure effects of different ketogenic diets and as such a myriad of hypotheses have been generated for the mechanisms of action [39-42]. Proposed mechanisms behind this dietary intervention include preventing neuronal hyperexcitability, direct inhibition of ion channels, influencing mitochondrial functions and altering amino acid metabolism to influence specific inhibitory neurotransmitter production [43-45].

At present, it is unclear whether the original KD would prove to be an effective anti-epileptic management option in dogs. To improve palatability and ketonemia, a variant of 'classic'KD was introduced in 1971 by Huttenlocher 
[46]. This subtype utilises medium chain triglycerides (MCT) as fat resource found as being more ketogenic than long chain triglycerides (LCT) [47]. Up to date, many research groups found that MCT-KD had also good antiseizure properties $[16,41,48]$. As dogs have a higher capacity for ketone body utilisation, it is hard to induce and/or maintain clinically effective levels of ketosis and anticonvulsant efficacy in dogs comparable to human medicine [49]. In contrast, MCTs are considered also as being more ketogenic in dogs due to subsequent transportation without pancreatic response and primarily metabolization into ketone bodies in the liver [39, 48-50]. MCT-KD came therefrom more into research focus as the ketogenic diet for $\operatorname{dogs}[23,24,49,51]$.

The main constituent of MCT-KD are normally octanoic (C8, caprylic) and decanoic (C10, capric) triglycerides (TAGs) [52, 53]. A recent in-vitro study has revealed decanoic acid acts as a non-competitive AMPA receptor antagonist that results in direct inhibition of excitatory neurotransmission, and thus exerts an anticonvulsant effect [54]. In comparison, octanoic acid alone revealed no anti-epileptic effects in in-vitro studies [55], but enhanced anticonvulsive properties in mice, when orally co- administered with C10 [56]. Ongoing research focusing on in vitro and rodent models also highlighted the importance of medium chain fatty acids with specific branching as anticonvulsant $[57,58]$.

In dogs, consumption of an MCT-enriched diet (10\% of total formula calories with $\mathrm{C} 8, \mathrm{C} 10, \mathrm{C} 12$ ) leads to significant elevation in BHB concentration and improved seizure control [24, 45, 59-61]. MCT intake also appears to be linked to a significant improvement in attentiondeficit hyperactivity disorder (ADHD) -related behaviour and fear/anxiety in dogs with epilepsy [23] and to have cognition-improving effects in aged dogs [61]. Recent research confirmed the observed benefits from MCT enriched kibble diet in aged dogs with cognitive dysfunction syndrome (CDS) in a prospective double blinded placebo controlled study design [62].

In conclusion, albeit past research provides a solid base, the exact metabolic mechanisms governing antiseizure effects from BHB and/or MCT remain elusive, not only in humans, but especially in dogs. Whether MCTs can be an effective anti-seizure agent and show neuroprotective and anxiolytic effects in dogs with IE requires further investigation.

We hypothesise MCTs administered as a daily supplement will reduce seizure frequency and severity, reduce the level of ASD side effects and positively influence behavioural comorbidities of epilepsy in dogs. The primary aims of this clinical study are to investigate the short- term influence of MCTs given as a DS on seizure occurrence, disease progression, drug-response, behaviour and QoL of dogs with IE. Twelve weeks of feeding is considered the minimal duration to allow short-term evaluation of efficacy and safety for diets $[24,59]$. Using the same test system as in our former study which for the first time demonstrated the efficacy of an MCT- enriched kibble diet [24] will help us draw comparisons between studies and provide results in a reasonable time frame.

The rationale of the current study is not only to corroborate the former results [4, 63] using a supplement rather than using a complete manufactured diet, but also to investigate the broader effects of MCTs on epilepsy and its comorbidities including their effects on cognition and behavioural comorbidities. This study should provide further information about the role of MCT ingestion and its importance in canine epilepsy management $[48,49]$.

\section{Methods and study design}

\section{Recruitment}

Participants are informed about this trial through different media in the UK, Germany, Austria, Switzerland and Finland. For advertisement, individual flyers and websites are made supporting the recruitment process. Epilepsy phenotype is determined using previous medical history and a standardised clinical history questionnaire (Additional file 1). Throughout this study, all dogs are recruited by a two-step screening system. Initially, all interested owners are asked to complete an online pre-study questionnaire capturing important data about the signalment, history, clinical appearance, diagnostics and treatment of their dog's IE. All applications are individually screened and selected in batches based on defined criteria and details then send to each study site.

\section{Study design}

A 6-month randomised, double-blinded, prospective, multicentre, dietary crossover trial is designed to compare two oil-DSs (MCT oil, control oil (olive oil)) in dogs with IE (Figs. 1 and 2). The experimental unit is each individual dog. Law et al., 2015 demonstrated a significant difference in 21 dogs completing the study, with a drop-out rate of approximately $30 \%$. In this study, we aim to enrol a total of thirty-six (36) dogs between five different study sites in three different countries in Europe (UK: Royal Veterinary College and, Pride Veterinary Centre, Germany: Tierarztpraxis Strassenheim, Tierarztpraxis Bathen-Nöthen and, Tierärztliche Hochschule Hanover, Finland: University Helsinki).

The monitoring site for this trial is the Clinical Investigation Centre (CIC) of the Royal Veterinary College (RVC), which oversees the logistical progression of the study under the supervision of the Study Monitor and leader. This study is conducted in accordance with veterinary GCP guidelines ((i) International Cooperation on Harmonisation of Technical Requirements for Registration of Veterinary Products 


\begin{tabular}{|c|c|c|c|c|c|c|c|c|}
\hline & \multicolumn{8}{|c|}{ STUDY PERIOD } \\
\hline & \multirow{2}{*}{$\begin{array}{c}\begin{array}{c}\text { Pre- } \\
\text { Enrolment }\end{array} \\
-V 1\end{array}$} & \multirow{2}{*}{$\begin{array}{c}\begin{array}{c}\text { Enrol- } \\
\text { ment }\end{array} \\
-V 1\end{array}$} & \multirow{2}{*}{$\frac{\text { Allocation }}{V 1}$} & \multicolumn{4}{|c|}{ Post-allocation } & \multirow{2}{*}{$\frac{\text { Close-ou }}{\text { V3 }}$} \\
\hline TIMEPOINT & & & & $T C 1$ & $V 2$ & $T C 2$ & V3 & \\
\hline \multicolumn{9}{|l|}{ ENROLMENT: } \\
\hline Eligibility screen & $\mathrm{X}$ & & & & & & & \\
\hline \multirow{2}{*}{$\begin{array}{r}\text { Informed consent } \\
\text { Allocation: Block } \\
\text { randomization }\end{array}$} & & $\mathrm{X}$ & & & & & & \\
\hline & & & $\mathrm{X}$ & & $\mathrm{X}$ & & & \\
\hline \multicolumn{9}{|l|}{ INTERVENTIONS: } \\
\hline $\begin{array}{r}\text { [Intervention: } A B \\
M C T-D S=1 s t]\end{array}$ & & & & $\rightarrow$ & & -- & -- & -- \\
\hline $\begin{array}{r}\text { [Intervention } B A, \\
M C T-D S=2 n d]\end{array}$ & & & $\leftarrow--$ & $-\rightarrow$ & $\leftarrow$ & & & $\rightarrow$ \\
\hline \multicolumn{9}{|l|}{ ASSESSMENTS: } \\
\hline $\begin{array}{r}\text { [Baseline } \text { : Body } \\
\text { weight, age, body } \\
\text { temperature } \\
\text { examination, drug } \\
\text { serum concentration] }\end{array}$ & & $\mathrm{X}$ & & $(\mathrm{X})$ & $\mathrm{X}$ & $(\mathrm{X})$ & $\mathrm{X}$ & $\mathrm{X}$ \\
\hline $\begin{array}{r}\text { [Outcome: } \\
\text { Examination, Seizure } \\
\text { data, beta- } \\
\text { hydroxybutyrate, bile } \\
\text { acids stimulation test, } \\
\text { VAS scoring } \\
\text { metabolome, } \\
\text { microbiome] }\end{array}$ & & $\mathrm{X}$ & & & $\mathrm{X}$ & & $\mathrm{X}$ & $\mathrm{X}$ \\
\hline $\begin{array}{r}\text { [List other data } \\
\text { variables: hematology, } \\
\text { biochemistry, VAS, } \\
\text { behavior testing, } \\
\text { questionnaire data, gait } \\
\text { analysis] }\end{array}$ & & $\mathrm{X}$ & & & $\mathrm{X}$ & & $\mathrm{X}$ & $\mathrm{X}$ \\
\hline
\end{tabular}

Fig. 1 SPIRIT aligned schedule of enrolment, interventions, and assessments

(VICH) GL9 Good Clinical Practices (July, 2001), (ii) The European Agency for the Evaluation of Medicinal Products (EMEA) Guideline on statistical principles for veterinary clinical trials, EMEA/CVMP/816/00-FINAL (June 5, 2002)). The study is approved by the Clinical Research Ethical Review Board (URN 2016 1558).

Each dog is randomly assigned using block randomisation to begin their first dietary period with either MCT or the placebo DS. The owner and investigators will not be informed of diet group assignment for each dog. All recruited dogs will be fed initially for $90 \pm 2$ days with either the control DS or the MCT DS alongside their normal diet, followed by $97 \pm 2$ days with the other supplement alongside their normal diet. The second feeding period includes the first week as a wash out period, which will not be considered in the statistical analysis. Each dog is evaluated on-site at the enrolment visit (-V1), and at the end of each $90 \pm 2$-day or 2 nd $97 \pm 2$ dietary period (V2 $=90 \pm 2$ days, V3 $=187 \pm 2$ days). Between on-site visits, owners will be contacted by phone to check for any difficulties arisen during the study period and to ensure compliance ( $\mathrm{TC} 1=45 \pm 2, \mathrm{TC} 2=142 \pm 2$ days).

For all trial participants, the dog should be fed twice daily with their regular diet, with the half amount of the total daily oil requirement. A total of $9 \%$ of the daily metabolic energy per day is from the oil. ASD treatment will not be withheld at any point nor changed in this study, however, owners will be allowed to stop their participation in the trial at any time point, as requested by the Clinical Research Ethical Review Board. Dogs that have been receiving other oils or oil type DS previously can take part in this study when there has been a pre-wash out period of at least 14 days before visit 1 . All owners are asked to keep the diet and drug treatment constant throughout the study period and to document any adverse events. 




Fig. 2 Study Design A 6-month randomised, double-blinded, prospective, multicentre, dietary crossover trial is designed to compare two oil dietary supplements (MCT oil, Control oil (=olive oil)). Both oils are given as $9 \%$ of their daily metabolic energy requirements. All dogs are aimed to be fed initially for $90+/-2$ days with either the control dietary supplement or the MCT dietary supplement alongside their normal diet followed by $97+/-2$ days (including initial one-week wash-out period, 7 days) with the other supplement alongside their normal diet. Another follow-up study about the long-term effects of MCT oil as an open-label use is subsequently planned after the individual patient has completed the main clinical trial

The 'Animal Research: Reporting in-Vivo Experiments' (ARRIVE) [64] and the Schedule Of Enrolment, Interventions, And Assessments (SPIRIT) reporting guidelines [65] have been considered and integrated into this study protocol were applicable (see also Additional file 2) . 


\section{Investigational animals - study phenotype}

Only dogs that satisfy all of the inclusion criteria and to whom none of the exclusion criteria apply will be enrolled and receive at enrolment a unique study number for identification purposes. All owners must give written informed consent to participate in the study and agree to fulfil all requirements of the study. All mixed or pure-breed dogs participating in this study are privately owned. The pet dogs should be at least 6 months of age on Day 0 and weigh at least $4 \mathrm{~kg}$. To be recruited, dogs must be in general good health and have an unremarkable interictal neurological examination for a dog on ASD treatment. Additionally, each dog must have met in general the International Veterinary Epilepsy Task Force (IVETF) tier II confidence level diagnosis of IE [4]. The current IVETF statement is, however, not yet clear about the importance of age in Tier II or above. In this study, dogs older than 6 years will be included as long they meet all the other inclusion criteria. Each dog will need to be treated with and resistant to least one ASD to be recruited. Drug-resistant patients are defined in line with the IVETF's definition of therapeutic success Category 3 [63]. In addition, each patient must have a required seizure frequency of at least three or more seizures in the last 3 months despite properly monitored pharmacotherapy prior to enrolment. Any dog with a known cause of epilepsy (structural epilepsy) e.g. brain neoplasm, brain trauma, encephalitis and meningitis are excluded. Any acute medical or surgical condition, pregnancy, lactation, history of pancreatitis or history of chronic or acute renal, hepatic or cardiac failure would lead to the patient's exclusion. Furthermore, dogs on drugs, diets or DS which could influence the metabolism of phenobarbitone, imepitoin or other ASD given during the trial are not included.

\section{Palatability}

Altering the diet characteristics with oil supplementation can be at the expense of palatability and influence a dog's eating behaviour. The mean food intake, as a percentage, can be calculated for each period and used to assess the dogs eating preference. An intake ratio (IR) can represent consumption rates. In this clinical trial successful food intake is essential. A small pilot study will be conducted in advance to ensure palatability and comparability of oils used to guarantee overall diet acceptance.

\section{Baseline diet}

The baseline diet will be nutritionally assessed for each patient, as its composition might amplify or influence the anti-seizure properties of MCTs. This includes the individual feeding type (commercial vs. home-made; subtypes) and utilised feed components (brand, formulation and type). This information is then used to create an average main nutrient profile on a dry matter basis (MNP: crude protein, crude fat, crude ash, crude fibres, nitrogen free extracts (Nfe), moisture). For this purpose, analytical data will be requested from each company. If available, the inclusion of additional data from other relevant nutrients, such as minerals, vitamins or fatty acids, will be considered. The hypothesised anti-seizure effects from MCT oil supplementation should be then critically discussed in the light of the patients' whole diet.

\section{Investigational veterinary dietary supplement}

The details of the ingredients and the maintenance of each oil are stored in the Dispenser File at the monitoring site. The MCT DS used will only contain C8 and $\mathrm{C} 10$ in a certain ratio and therefrom allow the investigators to extend the knowledge about the role of the single medium-chain fatty acids (MCFAs) in the former publications used for cognition-enhancement in aged dogs [61] and in dogs with IE [24, 59]. Both oil DS will be commercially available. The DS will be then supplied by the Study Monitor to the Investigator and will be stored at room temperature in a locked room at each test site. The dispenser educates the owner to keep the oil administration consistent throughout the study period. From enrolment onwards, the owner is not allowed to change or give any additional foods apart from the usual diet, or other food supplementations during the study period.

\section{Study procedures}

As aforementioned, the owner is in contact with the investigator on the following occasions with a tolerance of \pm 2 days: enrolment visit (-V1) before the start of the first dietary intervention period 1 (V1)); Day 45 (telephone call 1 (TC1)); Day 90 (visit 2 (V2), before the crossover and start of the second dietary intervention period II); Day 142 (telephone call 2 ((TC2)), Day 187 for study completion (visit 3 (V3)).

For each on-site study visit, the investigator completes the following tasks (a-e) to collect relevant data and monitor the clinical progress of each patient, while other parameter will only be collected depending on local conditions ( $\mathrm{f}-\mathrm{i})$ :

(a) Body weight and temperature is recorded;

(b) Clinical and neurological examination is performed;

(c) Checks the owners' documentation of seizure occurrences by frequency, severity and subtypes using a standardised logbook.

(d) Visual analogue score to measure level of sedation, ataxia and quality of life;

(e) Blood samples for routine haematology, serum biochemistry, thyroid profile, canine pancreatic lipase (CPLI), beta-hydroxybutyrate (BHB), serum 
concentration of phenobarbitone and/or potassium bromide and dynamic bile acid test are taken;

(f) Saliva is collected in the morning of each study visit;

(g) Fresh urine and faeces are collected;

(h) Behavioural tests on anxiety and cognition are conducted;

(i) Gait measurements are conducted.

In order to assess the dog's behaviour and QoL between the visits, a questionnaire is completed by the owners, at the earliest $24 \mathrm{~h}$ in advance of the scheduled study appointment (Additional file 3). The questionnaire consists of five existing questionnaires covering multiple aspects of behaviour, cognition, ADHD-related behaviour, appetite and QoL:

(1) Canine Behavioral Assessment \& Research Questionnaire (C-BARQ) [66]

(2) Attention deficit hyperactivity disorder rating scale (ADHD-RS) [67]

(3) Canine cognitive dysfunction rating scale (CCDR) [68]

(4) Dog Obesity Risk and Appetite Questionnaire (DORA) [69]

(5) Evaluation of Quality of Life in Dogs with IE (EpiQoL) [70].

All concomitant treatments and medications including vaccines, anthelmintics and drugs for parasite prophylaxis for the patient are recorded by the Investigator on each contact with the owner and is to be included in parameter analysis. Any abnormal health observation that is unfavourable and unintended and occurs after an animal has been enrolled and fed with the dietary supplement, regardless of whether it is considered to be product-related, is an adverse event. The documentation of adverse events includes the following details: a description of the adverse event; length of the adverse event recorded in days; severity of the adverse event recorded using $1=$ mild, $2=$ moderate, $3=$ severe and $4=$ serious; frequency of the adverse event measured using $1=$ once, 2 = occasionally, 3 = regularly and $4=$ ongoing; concomitant treatment noted as $1=$ none and $2=$ yes; and final outcomes consisted of $1=$ resolved without further effects, $2=$ resolved with further effects, $3=$ unchanged, $4=$ euthanasia and $5=$ death. In reference to the study objectives, seizure occurrences during the study participation is not defined as an adverse event. Any concomitant treatments during the study period is recorded with details on indication, used products, given strength, doses and length of treatments. Concomitant changes of ASD medication or dosages throughout the study lead to study exclusion.
Specification of the Study Variables (Additional file 4: Table S1)

General physical and neurological examination and laboratory diagnostics

A routine inter-ictal clinical and neurological examination is performed at each study visit. All above mentioned blood samples are analysed and added to the patients file. Comparison of all blood parameters between the dietary intervention periods is planned.

\section{Seizure frequency, seizure days per months, inter-seizure interval}

The seizure frequency is recorded by the owners in the 'Seizure Diary' per dietary intervention period. Short-term efficacy will be assessed over a 3-month period. Only generalised seizures will be counted toward the efficacy measurement. The primary outcome parameter for seizure frequency is 'treatment success': Treatment success is defined as seizure freedom for a time-span exceeding three times the longest inter-seizure interval (days) for an individual dog considering the year preceding the study and for a minimum of 3 months (time to 1st seizure) based on owners' documentations. [63]. Consequently, patients with complete freedom of seizures or significant extension of the inter-seizure interval will be considered treatment success and treatment should thereafter be continued as open-label to assess the seizure free rate e.g. the percentage of patients with short-term or long-term freedom of seizures. Partial treatment success will only be assessed in this study for dogs, which will remain in the study for at least 3 months and have no change in treatment during this period. Partial therapeutic success is defined as a significant difference (reduction) in either: seizure frequency (seizures/month), seizure days frequency per months, seizure cluster days frequency per months, seizure severity measured by number of episodes of cluster seizures or status epilepticus during a 3-month period between the MCT and control periods. Finally, each dog will be classified as 'seizure free', if it experiences a $100 \%$ reduction in seizure frequency, and as a 'MCT responder' if it experiences at least $50 \%$ reduction in seizure frequency between both dietary periods.

\section{Seizure types}

Information from the standardized Seizure Diary will be used to define the type of seizures. Seizures will be classified according to the guidelines of IVETF [63]. In this study, generalised seizures are the main focus of interest, as they are better recognised by the owner and reported to their vet [71]. A generalised epileptic seizure is an epileptic seizure, which initial semiology indicates, or is consistent with, more than minimal involvement of both cerebral hemispheres. Generalised epileptic seizures are conceptualised as originating at some point within, and 
rapidly engaging, bilaterally distributed networks, vegetative symptoms and loss of consciousness. Focal epileptic seizures are conceptualised as originating within networks limited to one hemisphere. Cluster seizures are defined as an episode where more than one seizure occurs within a 24-h period [63]. Status epilepticus will be defined as (a) continuous seizure activity for more than 5 min or (b) two or more discrete epileptic seizures between which there is incomplete recovery of consciousness (for generalized convulsive seizures) [63].

\section{Behavioural comorbidities and cognition}

The conducted five questionnaires, the VAS scoring and the behavioural tests are used to evaluate the patients on neurological and behavioural changes in terms of appetite, cognition, trainability, anxiety, activity and ataxia at each visit of this trial. The questionnaires and behavioural tests will be completed during each of the three trial visits.

(a) Non-invasive behavioural tests:

- Anxiety testing [13, 72, 73]: Each dog will undergo two non-invasive tests to assess anxiety behaviour: (i) open field test (OFT); (ii) separation test (ST). The room setting including cameras will be standardised to ensure consistency between measurements. The recorded video clips will be retrospectively evaluated. Known anxietyassociated behaviours will be assessed in terms of frequency, intensity (likert-scale grading 1-10) and occurrence in percentage of overall time of experiment $[72,74]$.

- Cognition testing: Each dog will undergo two validated, non-invasive tests to measure different aspect of cognition (task 1: food searching, task 2: problem solving) as formerly described in dogs with and without epilepsy using the same scoring system $[75,76]$. The testing environment will be prepared for both tasks in a standardised empty room with no noise, smell or light distraction.

- Gait recording: At each visit the gait will be video recorded and analysed retrospectively. Fifty strides from a walking dog will be assessed and then used to quantify the level of ataxia as formerly described in detail $[77,78]$.

(b) Questionnaires: An alteration in individual calculated scores for the questionnaires between the visits indicates improvement or deterioration of variables measured. Calculated scores are compared between dietary periods, but also grouped by MCT responder rate and seizure control in order to elucidate associations and relationships. i. Canine Behavioral Assessment \& Research Questionnaire (C-BARQ) [66]: The cBARQ contains the following subscales used for future comparison between both dietary periods: trainability, stranger-directed fear, fear, ownerdirected fear, non-social fear, separation related fear, attachment and attention seeking, chasing, excitability and touch sensitivity. Findings will be also compared to other more-targeted questionnaires, as subsequently listed.

ii. Attention deficit hyperactivity disorder rating scale (ADHD-RS) [67]: ADHD-RS is a valid method to assess attention and activity skills in dogs. Predetermined from the 13 items, two subscales (inattention (6 items, [0-3]) and activity-impulsivity (7 items [0-3]) of the questionnaire are calculated and will be compared between both dietary periods. Multivariate analysis will also include age-, gender- and training-skills.

iii. Canine cognitive dysfunction rating scale (CCDR) [68]: Cognitive abilities will be rated on an owner-based report using the CCDR scoring system with 13 behavioural items. The score can help identify dogs which have clinical signs seen with canine cognitive dysfunction (CCD). A score of 0-39 is classified as normal, 40-49 at risk and $>50$ as CCD. Individual scores will be compared between study periods.

iv. Dog Obesity Risk and Appetite Questionnaire (DORA) [69]: A questionnaire of 34 items grouped into three dog and four owner factors is applied at each study visit to get reliable, owner reported measure of the dogs eating behaviour linked to each DS. Each subfactor is rated by option scaled questions and in numerical analysis transformed as a percentage of the maximum between 0 and 100 .

v. Evaluation of Quality of Life in Dogs with IE (EpiQoL) [70]: A questionnaire of 7 themes with 36 questions compromising subunits ("Seizure severity and frequency", "Adverse effects of antiepileptic drug (AED)", "Restrictions on the carer's life", "Frustrations over caring for a dog with IE", "Carer distaste of AED adverse effects", "Carer anxiety around the seizure event", "Perceptions on rectal diazepam use") are option scaled rated by the owner and compared in frequency.

(c) VAS: At each study visit the owner is asked to complete a VAS for ataxia, sedation and overall QoL using a line from 0 to $100 \mathrm{~mm}(0-100 \%)$. A secondary intersecting line that presented best the subjective severity should be set by the owner perpendicularly on a provided measurement line. A 
perpendicular line at $0 \mathrm{~mm}$ represents 'asymptomatic/normal', while at $100 \mathrm{~mm}$ either the dog shows 'ataxia so severe that it is unable to walk' or 'sedation to the extent that it only sleeps' or 'QoL is so poor euthanasia is requested'. On analysis, the length from 0 to the perpendicular line will be normalised to baseline for statistical comparison into a percentage value $(0-100 \%$, negative $=$ improvement, positive $=$ deterioration $)$.

\section{Monitoring metabolic responses}

At each study visit, blood is collected by venepuncture using standardised operation procedures for haematology, serum biochemistry, ASD serum concentrations (phenobarbital, potassium bromide), cPLI, BHB and dynamic bile acid testing. The BHB and postprandial bile acid sample is taken as one sample $2 \mathrm{~h}$ after feeding with oil supplement. The cortisol level in saliva will be assessed between study visits [79]. The samples will be collected at the same time in the morning of each study visit. Furthermore, serum, urine and faecal samples are collected. These samples are used to investigate the metabolome and microbiome, respectively [80].

\section{Data analysis}

After study completion, the data will be collated, and statistical analysis will be performed using SPPS V24 (IBM Deutschland GmbH, Ehningen, Germany) and Prism ${ }^{\circ}$ (GraphPad Software, San Diego, USA). Results will be considered significant if after correction for multiple testing the $p$ value $<0.05$.

For all baseline, efficacy and safety parameters, the following analysis will be performed (Additional file 4: Table S1):

- Individual values will be listed and if appropriate graphically presented per time point for each group.

- For the continuous parameters, descriptive statistics will be calculated and tabulated per time point for each group. If data are normally distributed (as ascertained via plotting histograms of relevant variables and using Kolmogorov-Smirnov test), the mean and standard error will be plotted over time for each group.

- For the categorical or discrete parameters, a frequency table will be calculated and tabulated per time point of each group. A graphical presentation over time for each group will be made by means of a bar or pie chart.

Comparisons between the DS groups are made using match-paired Student's t-tests for parametric data and Wilcoxon matched-pairs signed rank test for non-parametric data. The relationships between two variables are analysed using Pearson's correlation coefficient for parametric and Spearman test for non-parametric data. Optionally, further multiple comparison correction based will be applied on individual data sets. The presence of cluster seizures is compared between DS periods using the McNemar test.

Statistical analysis is planned to be a combination of univariate (UVA) and multivariate data analyses (MVA). A mixed effect model might be considered. Statistical testing will be adapted were appropriate (Additional file 4: Table S1).

\section{Power calculation}

Our previous study did indicate that the effect of MCTs on seizure frequency can be detected with $n=21$ dogs [24]. Similarly, another study suggested twenty-two dogs in each group being sufficient for showing significant differences between two diet groups for seizure frequency [51]. In conclusion, we aimed to recruit at least 36 dogs to reach a minimum of 22 dogs to anticipate a usual patient dropout-rate of $30 \%$.

\section{Discussion}

Using a crossover dietary trial approach, we will generate data to investigate the value of MCT oil supplementation as a management tool for dogs diagnosed with IE not responding to standard $\mathrm{ASD}(\mathrm{s})$. If clinically and statistically significant effects are observed between the dietary periods, MCT oil has the potential to become an easily adjustable add-on therapy for dogs with epilepsy.

This study will utilise a prospective, double-blinded randomised placebo-controlled trial with a crossover design. Considering these specific study features in the design, we do not only follow international high quality standards for clinical research, but also address potential biasing effects in clinical veterinary trials $[81,82]$. The integration of a dietary crossover with placebo/control period, double-blinding and block randomisation should minimise the regression-to-the-mean, the placebo and observer effects seen in other canine epilepsy trial in veterinary medicine [83]. We are confident that this design significantly increases the accuracy and representative nature of our expected findings on the use of MCT oil as nutritional add-on management in dogs with IE.

Regression-to-the mean arises often in clinical trials when a variable is extreme on its first, but closer to the mean on its second measurement or vice versa [84-87]. In canine epilepsy trials, usually an extreme (hardest-to-treat) group is selected from an epileptic dog population based on the measurement of a particular variable. In this study, only dogs with idiopathic epilepsy TIER 2 level, classified as 'drug-resistant' with a poor seizure control are recruited, so that this phenomenon must be addressed in the study design. If this effect is not considered, the clinical trial or the interpretation of our obtained data can 
lead to incorrect conclusions, so must be then expected to be inaccurate or incorrect [88] .

The placebo effect is a well-known phenomenon in human medicine [89], however, limited information about the placebo effect exists in veterinary medicine to date [90]. Reports so far described caregiver placebo effect especially in association to chronic anti-inflammatory or analgesic treatment in small animals [91-93]. A decrease in seizure frequency of around 30\% compared to baseline has been noted in different trials in canine epilepsy as potential placebo effects [83]. The occurrence of placebo effects are significantly associated with response expectancy [89]. In canine epilepsy trials, the expectation from owners is usually higher due to physiological stress, often poor seizure control and the continuous negative impact on their own QoL $[70,79,94,95]$ so that a notably subjective influence on the dog can be more likely. A general run-in period given by the recruitment process, where owners are first enrolled on a waiting list and their dog are checked on study criteria before giving the first treatment, will also support to mitigate the response expectancy.

Another impact can derive from the Hawthorne effect or also known as the observer effect. When study individuals are aware of being observed within a clinically trial, their response to the trial situation can not only modify, often falsify study variables. The Hawthorne effect can therefore also be seen as an pre-placebo expectation [96]. Alike in epilepsy research in human medicine [97], such type of reactivity should be considered as influencing factors from owners and their dogs in the design of canine epilepsy trials.

The integration of a dietary crossover in the mid of observation time, allows each participant to receive both dietary interventions. Each study object will therefore serve as its own control and reduce significant biasing effects [98]. Even though the retrospective baseline is documented, the data will be completely disregarded in most of the analysis and only applied were appropriate. This exclusion is to minimise the regression-to-the-mean and observed placebo effect in other canine epilepsy trial in veterinary medicine [83].

Keeping all involved individuals (tester, subject, owners) blind from the used dietary supplement is another attempt to eliminate bias and the expectancy from owners seeing responses in their dog's seizure control after giving the specific DS. Not only observer, but also investigator bias might otherwise provoke incorrect observations, so that using a double-blinded design is most appropriate here. Describing the blinding methodology is important to give veterinarians the ability to assess the validity of the test results and apply in clinical environment [99] .

A core principal for clinical trials in human and veterinary medicine is the randomisation $[100,101]$. The process of randomised allocation of each dog on its day of enrolment is considered in this study as being essential. The random assignment of each study subject to a predefined block (1st DS (AB), 2nd DS (BA)) reduces the impact of selection bias and increases the validity of the expected study results. Furthermore, the detailed reports about the allocation process makes it possible for practitioners to critically review the impact of bias on study results and make informed decisions regarding the treatment of their own epileptic patients [102].

Overall, using not only a crossover design with a placebo/ control period, but also a block randomisation protocol and blinding should limit all above mentioned biasing effects to a possible maximum. Acting against hypothetical, multi-sided bias by implementing described trial features, should enable us to collect and assume not only controlled, but also representative data about MCT consumption in dogs diagnosed with IE.

Dropout is often used as an outcome measure in clinical trials. But, in randomised clinical trial with epileptic patients, it appears to be higher than other clinical studies and partly explained by trial design features thus providing direction for future trial designs [103]. As a preventive measure, the total amount of patients recruited will be aimed to be increased by $>30 \%$ to ensure a sufficient number of patients that will finish the trial. Law et al. (2015) have shown an effect with 21 dogs finishing the trial. Furthermore, in another study on ketogenic diet, twenty-two dogs in each group were suggested to be sufficient for showing significant differences between two diet groups for seizure frequency [51]. Accordingly, we aimed to recruit at least 36 dogs to reach a minimum of 22 dogs factoring a usual dropout-rate of $30 \%$.

Although study features will be implemented to prevent massive biasing effects, limitations are in any clinical trial and must be reflected critically on data analysis. One limitation of this study design is that an intention to treat analysis (ITTA) can be challenging or impossible to perform. ITTA is an approach to the analysis in which our recruited patients are evaluated as block randomised, regardless of the dietary intervention they actually received [104]. This is especially hard to realise when most or all dogs that dropout are within the first study period, as was the case in our former MCT study [24]. There is a chance that if randomisation is not carried out carefully, that results could be skewed as study dropouts will not be included in the paired statistical analysis. It is therefore of pivotal importance to document in detail (and if possible, compare statistically) the reasons why owners withdraw their dog from the study and during which study period. As aforementioned in the last study [24], the majority of the dropout dogs (7 out of 10) did not complete the first respective randomised allocated diet, hence there was no outcome measure at all for the 
remaining respective diet group. Using the last observation carried forward is not possible as there was no observation for the second diet.

Furthermore, utilising a statistical imputation method of the outcome measure would require profound assumptions to be made on the missing outcome. Statistical imputation of outcome assuming the MCT and placebo diet would be the same introduces bias towards the null. On the other hand, imputation assuming a difference on the probability of the outcome introduces bias towards the treatment effect. Considering that the primary aim of the study was to determine the anti-seizure efficacy of the ketogenic MCT diet, the utilising of a per protocol analysis method is more suitable and logical [82].

Oil supplementation for dog owners as additional treatment for general health or targeted to epilepsy management is a common practice [105]. As the clinical trial aims to prove ketogenic MCT oil supplementation as nutritional management for epileptic dogs, dietary characteristics and acceptance must be considered. As a variable, palatability is the measure of intake of a food that indicates acceptance, or the measure of preference of one food over another [55]. Food with different characteristics can alter voluntary food intake by affecting palatability, taste or flavour [56]. Excluding bias linked to palatability in the main clinical trial, the food intake ratio should be similar. The mean food intake, as a percentage, can be calculated for each period and was used to assess the dogs eating preference. An intake ratio (IR) of over 0.50 would represent equivalent consumption. If intake ratio (IR) for a feeding period was greater than 0.50 at 0.55 , this would lead one to conclude that it was consumed at a greater quantity [61]. Both oils will be proven in advance in a small palatability study to be well tolerated, similar consumed and no food preference was observed. Eating time might be increased when the oil DS is given, and this may be due to 'neophobia' [57] - and this may take time for the dogs to habituate to the presence of a new flavour/texture in their diet [58]. In summary, a good and similar palatability without any acute gastrointestinal side effects should be concluded and monitored in the prospective dietary trial.

Canine nutrition needs to be considered for epilepsy management [60, 107-110]. Diet composition, content and some of the DSs mentioned could influence pharmacokinetic properties of ASDs and potentially modulate their efficacy $[39,61]$. Owners of dogs with more severe epilepsy phenotypes, as recruited for this study, are likely to change unconsciously dietary regimes or DS due to higher levels of psychological stress $[70,79,106]$, therefore owners were asked to keep diets and DS constant throughout the trial. In addition, owners were asked to daily monitor any changes in seizures, drugs used, and potential adverse events seen. Nevertheless, a balanced supply of all nutrients is routinely not checked in first opinion practice and might play a role for the interpretation of dietary effects occurring from MCT oil supplementation. We will take great care of assessing the baseline diet as aforementioned. However, each dog will have a different baseline diet, which might influence the results and could be seen as another limitation of the study.

\section{Additional files}

Additional file 1: Pre-Study Questionnaire. Online survey designed for initial registration for the trial and to assess potential suitability of test candidate. (PDF $346 \mathrm{~kb}$ )

Additional file 2: SPIRIT Item Checklist (PDF 108 kb)

Additional file 3: Study Visit Questionnaire. Designed study questionnaire using five scientific validated questionnaire covering cognition, ADHD related behaviour, appetite and quality of life; Canine Behavioral Assessment \& Research Questionnaire (C-BARQ, Attention deficit hyperactivity disorder Questionnaire (ADHD) [67], The canine cognitive dysfunction rating scale (CCDR), Dog Obesity Risk and Appetite Questionnaire (DORA), Evaluation of Quality of Life in Dogs with Idiopathic Epilepsy (EpiQoL) to comparing dietary effects from both dietary supplements on behaviour in dogs diagnosed with TIER2 idiopathic epilepsy. (PDF 2596 kb)

Additional file 4: Table S1. Study Variables: For each on-site study visit, relevant data are collected and monitored by the investigator on the clinical progress of each patient. Collected data will be later converted into predefined study variable assessing MCT as DS in comparison to the control DS. This table should provide a detailed overview, which study part will contribute to which variable by units and which statistical tests are planned to evaluate the findings on significant differences or associations. Basically, grouping will be conducted by dietary period (I vs. II), DS type (MCT vs. Control-DS), MCT responder rate (seizure frequency reduction of at least $50 \%$ or less) and seizure types (cluster seizure presence, history of status epilepticus). On evaluation of the questionnaire and behavioural test scores other relevant factors (age, weight, amount of ASD per day etc.) will be considered for grouping. (DOCX 31 kb)

\section{Abbreviations}

ARRIVE: Animal Research: Reporting in-Vivo Experiments; ASD: Anti-seizure drug; C10: Decanoic acid; C8: Octanoic acid; CDS: Cognitive dysfunction syndrome; CIC: Clinical investigation centre; CSS: Cluster Seizure Status; DHD: Attention-deficit/hyperactivity disorder; DS: Dietary supplement; HARP: Harmonized animal research reporting principles; ICLAS: International Council for Laboratory Animal Science; IE: Idiopathic epilepsy; ITTA: Intentionto-treat analysis; IVETF: International Veterinary Epilepsy Task Force; MBW: Metabolic body weight; MCFA: Medium chain fatty acids; MCT: Medium chain triacylglyceride; ME: Metabolic energy requirement; MNP: Main nutrient profile on a dry matter basis; QoL: Quality of Life; RER: Resting energy requirement; RVC: Royal Veterinary College

\section{Acknowledgements \\ The authors thank the AKC Canine Health Foundation for financial support and the clinical investigation centre at the Royal Veterinary College for overseeing Good Clinical Practice (GCP) protocol guidelines (VICH GL9) aligned planning of this clinical trial. Additionally, the authors are grateful to all the owners who applied and were recruited for this study, who took the time to apply via the pre-study questionnaire, and those who helped in its distribution. Furthermore, BAB would like to acknowledge the Hans-Böckler Foundation for funding during the entire research project.}

\section{Funding}

This study conduction is externally funded by the AKC Canine Health Foundation as grant to HV and RMAP. (Grant 2252: Investigating a Ketogenic Medium-Chain Triglyceride (MCT) Supplement for the Treatment of Drug- 
Resistant Canine Idiopathic Epilepsy and Its Behavioral Comorbidities). This study protocol was peer reviewed by the AKC Canine Health Foundation and $B A B$ was recipient of a merit-based doctoral scholarship from the HansBöckler Foundation funded by the Federal Ministry of Education and Research - Germany (BMBF) and TL has been as collaborative, postdoctoral researcher funded by BBSRC grant BB/P001874/1.

\section{Availability of data and materials}

The data collected in this trial are collated and stored at the Royal Veterinary College in London (RVC). All data are anonymised as appropriate and only used for analysis. All personal information are held and used in accordance with the Data Protection Act 1998 and will not be disclosed to any unauthorised person or body. The trial has ended and all data are currently being analysed and prepared for publication. Data sharing is not applicable to this article as no datasets are generated or analysed; here is only the study design described, the data will be published separately.

\section{Authors' contributions}

BAB compiled this manuscript. BAB, RMAP, TL and HV made substantial contributions to conception and design, or acquisition of data. All other authors, AW, ABN, TSJ, AK, AT and LP, have been involved in drafting the manuscript or revising it critically for important intellectual content. All authors have given final approval for the submitted version to be published and have agreed all to be personally accountable for the author's own contributions, ensured that questions related to the accuracy or integrity of any part of the work.

\section{Ethics approval and consent to participate}

The study protocol and design were approved by the Royal Veterinary College (RVC) Clinical Research Ethical Review Board (CRERB) and ethical approval has been granted (URN 2016 1558). All owners must give written informed consent to participate in the study and agree to fulfil all requirements of the study.

\section{Consent for publication}

Not applicable.

\section{Competing interests}

$B A B, R M A P, T L, A W, A B N, T S J, A K, A T$ and $L P$ - No financial and non-financial competing interests exist.

$\mathrm{HV}$ is the Section Editor of the 'Neurology and Neuroscience' Section of BMC Veterinary Research, they played no role in the handling of this manuscript or editorial decisions. HV has no other financial or non-financial competing interests to declare.

\section{Publisher's Note}

Springer Nature remains neutral with regard to jurisdictional claims in published maps and institutional affiliations.

\section{Author details}

'Department of Clinical Science and Services (CSS), Royal Veterinary College, Hatfield, UK. ${ }^{2}$ Tierarztpraxis Strassenheim: BrainCheck.Pet, Ortsstraße Mannheim, Germany. ${ }^{3}$ Pride Veterinary Centre, Neurology/Neurosurgery Service, Riverside Road, Pride Park, Derby, UK. ${ }^{4}$ Tierarztpraxis, Dr. A. Bathen-Nöthen, Hatzfeldstraße, Cologne, Germany. ${ }^{5}$ Department of Equine and Small Animal Medicine, Faculty of Veterinary Medicine, Helsinki, Finland. ${ }^{6}$ Klinik für Kleintiere, Stiftung Tierärztliche Hochschule Hannover, Bünteweg, Hanover, Germany. ${ }^{7}$ Department of Comparative Biomedical Sciences (CBS), Royal Veterinary College, Hatfield, UK.

\section{Received: 7 May 2019 Accepted: 14 May 2019}

Published online: 30 May 2019

\section{References}

1. Erlen A, Potschka H, Volk HA, Sauter-Louis C, O'Neill DG. Seizure occurrence in dogs under primary veterinary care in the UK: prevalence and risk factors. J Vet Intern Med. 2018;32(5):1665-76.

2. Heynold Y, Faissler D, Steffen F, Jaggy A. Clinical, epidemiological and treatment results of idiopathic epilepsy in 54 labrador retrievers: a long-term study. J Small Anim Pract. 1997;38(1):7-14.
3. Kearsley-Fleet L, O'Neill DG, Volk HA, Church DB, Brodbelt DC. Prevalence and risk factors for canine epilepsy of unknown origin in the UK. Vet Rec. 2013;172(13):338.

4. De Risio L, Bhatti S, Munana K, Penderis J, Stein V, Tipold A, Berendt $M$, Farqhuar R, Fischer A, Long $S$, et al. International veterinary epilepsy task force consensus proposal: diagnostic approach to epilepsy in dogs. BMC Vet Res. 2015;11:148.

5. Charalambous M, Shivapour SK, Brodbelt DC, Volk HA. Antiepileptic drugs' tolerability and safety--a systematic review and meta-analysis of adverse effects in dogs. BMC Vet Res. 2016;12:79.

6. Wessmann A, Volk HA, Packer RM, Ortega M, Anderson TJ. Quality-oflife aspects in idiopathic epilepsy in dogs. Vet Rec. 2016;179(9):229.

7. Munana KR. Management of refractory epilepsy. Top Companion Anim Med. 2013;28(2):67-71.

8. Podell M, Fenner WR. Bromide therapy in refractory canine idiopathic epilepsy. J Vet Intern Med. 1993;7(5):318-27.

9. Podell M. Antiepileptic drug therapy. Clin Tech Small Anim Pract. 1998;13(3): 185-92.

10. Trepanier LA, Van Schoick A, Schwark WS, Carrillo J. Therapeutic serum drug concentrations in epileptic dogs treated with potassium bromide alone or in combination with other anticonvulsants: 122 cases (1992-1996). J Am Vet Med Assoc. 1998;213(10):1449-53.

11. Schwartz-Porsche D, Loscher W, Frey HH. Therapeutic efficacy of phenobarbital and primidone in canine epilepsy: a comparison. J Vet Pharmacol Ther. 1985;8(2):113-9.

12. Packer RM, Shihab NK, Torres BB, Volk HA. Responses to successive anti-epileptic drugs in canine idiopathic epilepsy. Vet Rec. 2015; 176(8):203.

13. Packer RMA, Hobbs SL, Blackwell EJ. Behavioral interventions as an adjunctive treatment for canine epilepsy: a missing part of the epilepsy management toolkit? Frontiers in veterinary science. 2019;6:3.

14. Martle V, Van Ham L, Raedt R, Vonck K, Boon P, Bhatti S. Nonpharmacological treatment options for refractory epilepsy: an overview of human treatment modalities and their potential utility in dogs. Vet J. 2014; 199(3):332-9.

15. Larsen JA, Owens TJ, Fascetti AJ. Nutritional management of idiopathic epilepsy in dogs. J Am Vet Med Assoc. 2014;245(5):504-8.

16. Augustin K, Khabbush A, Williams S, Eaton S, Orford M, Cross JH, Heales SJR, Walker MC, Williams RSB. Mechanisms of action for the medium-chain triglyceride ketogenic diet in neurological and metabolic disorders. Lancet Neurol. 2018;17(1):84-93.

17. Wijnen BF, de Kinderen RJ, Lambrechts DA, Postulart D, Aldenkamp AP, Majoie MH, Evers SM. Long-term clinical outcomes and economic evaluation of the ketogenic diet versus care as usual in children and adolescents with intractable epilepsy. Epilepsy Res. 2017;132:91-9.

18. Patterson EE. Canine epilepsy: an underutilized model. ILAR J. 2014;55(1): $182-6$.

19. Achanta $L B$, Rae CD. Beta-Hydroxybutyrate in the brain: one molecule, multiple mechanisms. Neurochem Res. 2017:42(1):35-49.

20. Lusardi TA, Akula KK, Coffman SQ, Ruskin DN, Masino SA, Boison D. Ketogenic diet prevents epileptogenesis and disease progression in adult mice and rats. Neuropharmacology. 2015;99:500-9.

21. Mantis JG, Meidenbauer JJ, Zimick NC, Centeno NA, Seyfried TN. Glucose reduces the anticonvulsant effects of the ketogenic diet in EL mice. Epilepsy Res. 2014;108(7):1137-44.

22. Bosch G, Beerda B, Hendriks WH, van der Poel AF, Verstegen MW. Impact of nutrition on canine behaviour: current status and possible mechanisms. Nutr Res Rev. 2007;20(2):180-94.

23. Packer RM, Law TH, Davies E, Zanghi B, Pan Y, Volk HA. Effects of a ketogenic diet on ADHD-like behavior in dogs with idiopathic epilepsy. Epilepsy Behav. 2016;55:62-8.

24. Law TH, Davies ES, Pan Y, Zanghi B, Want E, Volk HA. A randomised trial of a medium-chain TAG diet as treatment for dogs with idiopathic epilepsy. Br J Nutr. 2015;1 14(9):1438-47.

25. Masino SA, Freedgood NR, Reichert HR, Director CJ, Whittemore VH, ZupecKania B. Dietary intervention for canine epilepsy: two case reports. Epilepsia Open. 2019;4(1):193-9.

26. Lujan ASS, Anderson TK. The role of diet in refractory canine epilepsy - a retrospective case series [abstract]. In: BSAVA Congress 2004: Scientific Proceedings: 2004. Quedgeley: British small animal veterinary association; 2004. p. 53. 
27. Geyelin HR. Fasting as a method for treating epilepsy. Med Rec. 1921; 99:1037-9.

28. Laffel L. Ketone bodies: a review of physiology, pathophysiology and application of monitoring to diabetes. Diabetes Metab Res Rev. 1999; 15(6):412-26.

29. Freeman JM, Kossoff EH, Hartman AL. The ketogenic diet: one decade later. Pediatrics. 2007;119(3):535-43.

30. Likhodii SS, Serbanescu I, Cortez MA, Murphy P, Snead OC 3rd, Burnham WM. Anticonvulsant properties of acetone, a brain ketone elevated by the ketogenic diet. Ann Neurol. 2003;54(2):219-26.

31. Li J, O'Leary El, Tanner GR. The ketogenic diet metabolite betahydroxybutyrate (beta-HB) reduces incidence of seizure-like activity (SLA) in a Katp- and GABAb-dependent manner in a whole-animal Drosophila melanogaster model. Epilepsy Res. 2017;133:6-9.

32. Si J, Wang S, Liu N, Yang X, Wang Y, Li L, Wang J, Lv X. Anticonvulsant effect of exogenous beta-hydroxybutyrate on kainic acid-induced epilepsy. Exp Ther Med. 2017;14(1):765-70.

33. Hu E, Du H, Zhu X, Wang L, Shang S, Wu X, Lu H, Lu X. Beta-hydroxybutyrate promotes the expression of BDNF in hippocampal neurons under adequate glucose supply. Neuroscience. 2018;386:315-25.

34. Rho JM, Anderson GD, Donevan SD, White HS. Acetoacetate, acetone, and dibenzylamine (a contaminant in I-(+)-betahydroxybutyrate) exhibit direct anticonvulsant actions in vivo. Epilepsia. 2002;43(4):358-61.

35. Simeone TA, Simeone KA, Rho JM. Ketone Bodies as Anti-Seizure Agents. Neurochem Res. 2017:42(7):2011-8.

36. Simeone TA, Simeone KA, Stafstrom CE, Rho JM. Do ketone bodies mediate the anti-seizure effects of the ketogenic diet. Neuropharmacology. 2018;133: 233-41.

37. Kadowaki A, Sada N, Juge N, Wakasa A, Moriyama Y, Inoue T. Neuronal inhibition and seizure suppression by acetoacetate and its analog, 2phenylbutyrate. Epilepsia. 2017;58(5):845-57.

38. Zhu F, Shan W, Xu Q, Guo A, Wu J, Wang Q. Ketone bodies inhibit the opening of acid-sensing ion channels (ASICS) in rat hippocampal excitatory neurons in vitro. Front Neurol. 2019;10:155.

39. Sills MA, Forsythe WI, Haidukewych D, MacDonald A, Robinson M. The medium chain triglyceride diet and intractable epilepsy. Arch Dis Child. 1986;61(12):1168-72.

40. Trauner DA. Medium-chain triglyceride (MCT) diet in intractable seizure disorders. Neurology. 1985;35(2):237-8.

41. Liu YM. Medium-chain triglyceride (MCT) ketogenic therapy. Epilepsia. 2008; 49(Suppl 8):33-6.

42. Wheless JW. History of the ketogenic diet. Epilepsia. 2008;49(s8):3-5.

43. Clanton RM, Wu G, Akabani G, Aramayo R. Control of seizures by ketogenic diet-induced modulation of metabolic pathways. Amino Acids. 2016;49(1):120.

44. Baranano KW, Hartman AL. The ketogenic diet: uses in epilepsy and other neurologic illnesses. Curr Treat Options Neurol. 2008;10(6):410-9.

45. Law TH, Volk HA, Pan Y, Zanghi B, Want EJ. Metabolic perturbations associated with the consumption of a ketogenic medium-chain TAG diet in dogs with idiopathic epilepsy. Br J Nutr. 2018:120(5):484-90

46. Huttenlocher PR, Wilbourn AJ, Signore JM. Medium-chain triglycerides as a therapy for intractable childhood epilepsy. Neurology. 1971;21(11):1097-103.

47. Schon H, Lippach I, Gelpke W. Metabolic studies with a mixed glyceride of middle-chain fatty acids. II. Investigations on the changes of ketone-body content of blood \& urine after the administration of the mixed glycerides. Gastroenterologia. 1959;91(3):199-213.

48. Kelley SA, Hartman AL. Metabolic treatments for intractable epilepsy. Semin Pediatr Neurol. 2011;18(3):179-85.

49. Puchowicz MA, Smith CL, Bomont C, Koshy J, David F, Brunengraber H. Dog model of therapeutic ketosis induced by oral administration of R,S-1,3butanediol diacetoacetate. J Nutr Biochem. 2000;11(5):281-7.

50. James FE, Mansfield CS, Steiner JM, Williams DA, Robertson ID. Pancreatic response in healthy dogs fed diets of various fat compositions. Am J Vet Res. 2009;70(5):614-8.

51. Patterson EEMK, Kirk CA, et al. Results of a ketogenic food trial for dogs with idiopathic epilepsy. J Vet Intern Med. 2005;19:421.

52. Sills MA, Forsythe WI, Haidukewych D. Role of octanoic and decanoic acids in the control of seizures. Arch Dis Child. 1986;61(12):1173-7.

53. Kossoff EH. Chapter 83 - Nonpharmacological approaches: diet and neurostimulation. In: Dulac O, Lassonde M, Sarnat HB, editors. Handbook of
Clinical Neurology, vol. 111: Elsevier; Amsterdam; The Netherlands; eBook ISBN: 9780444626981; 2013. p. 803-8.

54. Chang P, Augustin K, Boddum K, Williams S, Sun M, Terschak JA, Hardege JD, Chen PE, Walker MC, Williams RS. Seizure control by decanoic acid through direct AMPA receptor inhibition. Brain. 2016;139(Pt 2):431-43.

55. Schuck PF, Ferreira Gda C, Tonin AM, Viegas CM, Busanello EN, Moura AP, Zanatta A, Klamt F, Wajner M. Evidence that the major metabolites accumulating in medium-chain acyl-CoA dehydrogenase deficiency disturb mitochondrial energy homeostasis in rat brain. Brain Res. 2009;1296:117-26.

56. Wlaz P, Socala K, Nieoczym D, Zarnowski T, Zarnowska I, Czuczwar SJ, Gasior M. Acute anticonvulsant effects of capric acid in seizure tests in mice. Prog Neuro-Psychopharmacol Biol Psychiatry. 2015;57:110-6.

57. Chang P, Terbach N, Plant N, Chen PE, Walker MC, Williams RS. Seizure control by ketogenic diet-associated medium chain fatty acids. Neuropharmacology. 2013;69:105-14.

58. Chang P, Zuckermann AM, Williams S, Close AJ, Cano-Jaimez M, McEvoy JP, Spencer J, Walker MC, Williams RS. Seizure control by derivatives of medium chain fatty acids associated with the ketogenic diet show novel branchingpoint structure for enhanced potency. J Pharmacol Exp Ther. 2015;352(1): 43-52.

59. Law TH, Davies ES, Pan Y, Zanghi B, Want E, Volk HA. A randomised trial of a medium-chain TAG diet as treatment for dogs with idiopathic epilepsy CORRIGENDUM. Br J Nutr. 2016;115(9):1696.

60. Gilbert DL, Pyzik PL, Freeman JM. The ketogenic diet: seizure control correlates better with serum beta-hydroxybutyrate than with urine ketones. J Child Neurol. 2000;15(12):787-90.

61. Pan Y, Larson B, Araujo JA, Lau W, de Rivera C, Santana R, Gore A, Milgram NW. Dietary supplementation with medium-chain TAG has long-lasting cognition-enhancing effects in aged dogs. Br J Nutr. 2010;103(12):1746-54

62. Pan Y, Landsberg G, Mougeot I, Kelly S, Xu H, Bhatnagar S, Gardner CL, Milgram NW. Efficacy of a therapeutic diet on dogs with signs of cognitive dysfunction syndrome (CDS): a prospective double blinded placebo controlled clinical study. Front Nutr. 2018:5:127.

63. Berendt M, Farquhar RG, Mandigers PJ, Pakozdy A, Bhatti SF, De Risio L, Fischer A, Long S, Matiasek K, Munana K, et al. International veterinary epilepsy task force consensus report on epilepsy definition, classification and terminology in companion animals. BMC Vet Res. 2015;11:182.

64. Osborne N, Avey MT, Anestidou L, Ritskes-Hoitinga M, Griffin G. Improving animal research reporting standards. HARRP, the first step of a unified approach by ICLAS to improve animal research reporting standards worldwide. EMBO Rep. 2018;19(5).

65. Chan A-W, Tetzlaff JM, Altman DG, Laupacis A, Gøtzsche PC, Krleža-Jerić K, Hróbjartsson A, Mann H, Dickersin K, Berlin JA, et al. SPIRIT 2013 statement: defining standard protocol items for clinical trials. Ann Intern Med. 2013; 158(3):200-7.

66. Hsu Y, Serpell JA. Development and validation of a questionnaire for measuring behavior and temperament traits in pet dogs. J Am Vet Med Assoc. 2003;223(9):1293-300.

67. Vas J, Topál J, Péch É, Miklósi Á. Measuring attention deficit and activity in dogs: a new application and validation of a human ADHD questionnaire. Appl Anim Behav Sci. 2007;103(1-2):105-17.

68. Salvin HE, McGreevy PD, Sachdev PS, Valenzuela MJ. The canine cognitive dysfunction rating scale (CCDR): a data-driven and ecologically relevant assessment tool. Vet J. 2011;188(3):331-6.

69. Raffan E, Smith SP, O'Rahilly S, Wardle J. Development, factor structure and application of the dog obesity risk and appetite (DORA) questionnaire. PeerJ. 2015:3:e1278.

70. Wessmann A, Volk HA, Parkin T, Ortega M, Anderson TJ. Evaluation of quality of life in dogs with idiopathic epilepsy. J Vet Intern Med. 2014;28(2): 510-4.

71. Packer RM, Lucas R, Volk HA. Owner perception of focal seizures in canine epilepsy. Vet Rec. 2017;180(6):150.

72. Gruen ME, Case BC, Foster ML, Lazarowski L, Fish RE, Landsberg G, DePuy V, Dorman DC, Sherman BL. The use of an open field model to assess soundinduced fear and anxiety associated behaviors in Labrador retrievers. J Vet Behav. 2015;10(4):338-45.

73. Ogata N. Separation anxiety in dogs: what progress has been made in our understanding of the most common behavioral problems in dogs. J Vet Behav. 2016;16:28-35.

74. Konok V, Dóka A, Miklósi Á. The behavior of the domestic dog (Canis familiaris) during separation from and Reunion with the owner: a 
questionnaire and an experimental study. Appl Anim Behav Sci. 2011;135(4): 300-8.

75. Gonzalez-Martinez A, Rosado B, Pesini P, Garcia-Belenguer S, Palacio J, Villegas A, Suarez ML, Santamarina G, Sarasa M. Effect of age and severity of cognitive dysfunction on two simple tasks in pet dogs. Vet J. 2013;198(1): 176-81.

76. Winter J, Packer RMA, Volk HA. Preliminary assessment of cognitive impairments in canine idiopathic epilepsy. Vet Rec. 2018;182(22):633.

77. Suiter EJ, Packer RM, Volk HA. Comparing the effects of first-line antiepileptic drugs on the gait of dogs with idiopathic epilepsy. Vet Rec. 2016;178(26):652.

78. Olsen E, Suiter EJ, Pfau T, McGonnell IM, Matiasek K, Giejda A, Volk HA Cavalier king Charles spaniels with Chiari-like malformation and Syringomyelia have increased variability of spatio-temporal gait characteristics. BMC Vet Res. 2017:13(1):159.

79. Packer RMA, Volk HA, Fowkes RC. Physiological reactivity to spontaneously occurring seizure activity in dogs with epilepsy and their carers. Physiol Behav. 2017;177:27-33

80. Schmidt M, Unterer S, Suchodolski JS, Honneffer JB, Guard BC, Lidbury JA, Steiner JM, Fritz J, Kolle P. The fecal microbiome and metabolome differs between dogs fed bones and raw food (BARF) diets and dogs fed commercial diets. PLoS One. 2018;13(8):e0201279.

81. Lund EM, James KM, Neaton JD. Clinical trial design: veterinary perspectives. J Vet Intern Med. 1994;8(5):317-22.

82. Oyama MA, Ellenberg SS, Shaw PA. Clinical trials in veterinary medicine: a new era brings new challenges. J Vet Intern Med. 2017;31(4):970-8.

83. Munana KR, Zhang D, Patterson EE. Placebo effect in canine epilepsy trials. J Vet Intern Med. 2010;24(1):166-70.

84. Stigler SM. Regression towards the mean, historically considered. Stat Methods Med Res. 1997;6(2):103-14.

85. Morton V, Torgerson DJ. Regression to the mean: treatment effect without the intervention. J Eval Clin Pract. 2005;11(1):59-65.

86. Bland JM, Altman DG. Regression towards the mean. Bmj. 1994;308(6942): 1499

87. Sjoberg RL. A critical question: therapeutic effect or regression towards the mean? Lakartidningen. 2006;103(26-27):2057-8.

88. Samuels ML. Statistical reversion toward the mean: more universal than regression toward the mean. Am Stat. 1991:45(4):344-6.

89. Whalley B, Hyland ME, Kirsch I. Consistency of the placebo effect. J Psychosom Res. 2008;64(5):537-41.

90. McMillan FD. The placebo effect in animals. J Am Vet Med Assoc. 1999; 215(7):992-9.

91. Conzemius MG, Evans RB. Caregiver placebo effect for dogs with lameness from osteoarthritis. J Am Vet Med Assoc. 2012;241(10):1314-9.

92. Gruen ME, Dorman DC, Lascelles BDX. Caregiver placebo effect in analgesic clinical trials for cats with naturally occurring degenerative joint diseaseassociated pain. Vet Rec. 2017;180(19):473.

93. Jaeger G, Larsen S, Moe L. Stratification, blinding and placebo effect in a randomized, double blind placebo-controlled clinical trial of gold bead implantation in dogs with hip dysplasia, vol. 46; 2005.

94. De Risio L, Freeman J, Shea A. Evaluation of quality of life of carers of Italian spinoni with idiopathic epilepsy. Vet Rec Open. 2016;3(1):e000174.

95. Packer RM, Volk HA. Epilepsy beyond seizures: a review of the impact of epilepsy and its comorbidities on health-related quality of life in dogs. Vet Rec. 2015;177(12):306-15.

96. Goldenholz DM, Goldenholz SR. Response to placebo in clinical epilepsy trials_old ideas and new insights. Epilepsy Res. 2016;122:15-25.

97. Goldenholz DM, Moss R, Scott J, Auh S, Theodore WH. Confusing placebo effect with natural history in epilepsy: a big data approach. Ann Neurol. 2015;78(3):329-36

98. Süt N. Study designs in medicine. Balkan Med J. 2014;31(4):273-7.

99. Giuffrida MA, Agnello KA, Brown DC. Blinding terminology used in reports of randomized controlled trials involving dogs and cats. J Am Vet Med Assoc. 2012;241(9):1221-6.

100. Gluud LL. Bias in clinical intervention research. Am J Epidemiol. 2006;163(6): 493-501.

101. Brown DC. Sources and handling of losses to follow-up in parallel-group randomized clinical trials in dogs and cats: 63 trials (2000-2005). Am J Vet Res. 2007:68(7):694-8.
102. Brown DC. Control of selection bias in parallel-group controlled clinical trials in dogs and cats: 97 trials (2000-2005). J Am Vet Med Assoc. 2006;229(6): 990-3.

103. Rabinowitz J, Levine SZ, Barkai O, Davidov O. Dropout rates in randomized clinical trials of antipsychotics: a meta-analysis comparing first- and secondgeneration drugs and an examination of the role of trial design features. Schizophr Bull. 2009;35(4):775-88.

104. Gravel J, Opatrny L, Shapiro S. The intention-to-treat approach in randomized controlled trials: are authors saying what they do and doing what they say? Clin Trials. 2007;4(4):350-6.

105. Berk BA, Packer RMA, Law TH, Volk HA. Investigating owner use of dietary supplements in dogs with idiopathic epilepsy. Res Vet Sci. 2018;119:276-84.

106. D'Aniello B, Semin GR, Alterisio A, Aria M, Scandurra A. Interspecies transmission of emotional information via chemosignals: from humans to dogs (Canis lupus familiaris). Anim Cogn. 2017:21(1):67-78.

107. Bontempo V. Nutrition and health of dogs and cats: evolution of petfood. Vet Res Commun. 2005;29(Suppl 2):45-50.

108. Manteca X. Nutrition and behavior in senior dogs. Top Companion Anim Med. 2011;26(1):33-6.

109. Matthews H, Granger N, Wood J, Skelly B. Effects of essential fatty acid supplementation in dogs with idiopathic epilepsy: a clinical trial. Vet J. 2012; 191(3):396-8.

110. Scorza FA, Cavalheiro EA, Arida RM, Terra VC, Scorza CA, Ribeiro MO, Cysneiros RM. Positive impact of omega-3 fatty acid supplementation in a dog with drug-resistant epilepsy: a case study. Epilepsy Behav. 2009;15(4): $527-8$.
Ready to submit your research? Choose BMC and benefit from:

- fast, convenient online submission

- thorough peer review by experienced researchers in your field

- rapid publication on acceptance

- support for research data, including large and complex data types

- gold Open Access which fosters wider collaboration and increased citations

- maximum visibility for your research: over $100 \mathrm{M}$ website views per year

At BMC, research is always in progress.

Learn more biomedcentral.com/submissions 